\title{
Instability of Poiseuille flow in a fluid overlying a porous layer
}

\author{
By MIN-HSING CHANG, FALIN CHEN \\ AND BRIAN STRAUGHAN \\ Department of Mechanical Engineering, Yung-Ta Institute of Technology and Commerce, \\ Ping Tung, Taiwan 909 \\ Institute of Applied Mechanics, National Taiwan University, Taipei, Taiwan 106 \\ Department of Mathematical Sciences, University of Durham, DH1 3LE, UK
}

(Received 11 February 2006 and in revised form 21 March 2006)

The problem of Poiseuille flow in a fluid overlying a porous medium saturated with the same fluid is studied. A careful linear instability analysis is carried out. It is shown that there are three modes of instability, two belong to one eigenvalue and persist in small ranges of parameters, while beyond these parameter ranges a third corresponding to another eigenvalue prevails. These three modes are of different stability characteristics, but are triggered by the shear stress of the Poiseuille flow in the fluid layer.

\section{Introduction}

The instability of two-dimensional parallel flows and in particular Poiseuille flow has been a major problem in fluid mechanics for many years. An excellent account of the early work on this problem may be found in Stuart (1963), or in Drazin \& Reid (1981, chap. 4). More recently, many writers have tried to explain discrepancies between the results of linear instability theory and experiments, see, for example, the optimal energy growth approach of Butler \& Farrell (1992). The latter work and related analysis involving energy stability theory is reviewed in Straughan (1998, chap. 8). The object of the present paper is to study the classical problem of Poiseuille flow, but when a Newtonian fluid overlies a layer of porous material saturated with the same fluid. We believe this is the first analysis of this problem.

The thermal convection problem for a fluid overlying a porous medium has been the object of much attention, cf. Chen \& Chen (1988), Nield (1998), Nield \& Bejan (1999) and Straughan (2001, 2002, 2004 p. 174). Chen \& Chen (1988) found that the linear instability curves for the onset of convection may be bi-modal. The parameter which is the key in their work is the depth ratio

$$
\hat{d}=\frac{d}{d_{m}}=\frac{\text { depth of fluid layer }}{\text { depth of porous layer }} .
$$

They find that for $\hat{d} \leqslant 0.13$, instability is initiated in and dominated by the porous medium, whereas for $\hat{d}$ greater than this value, the instability is effectively dominated by the fluid layer. Mathematically, this switching of fluid/porous layer roles manifests itself in having two maxima (or minima) on the neutral curve instead of the one found in the classical Bénard problem.

Flow in a porous-fluid system has numerous industrial and geotechnical applications and consequently has received much attention in the literature, cf. 
Nield (1983, 1991, 1998), Nield \& Bejan (1999), and the many references in the last contribution. In particular, Nield $(1983,1991,1998)$ has analysed in much detail two or more layer systems especially with regard to finding the most suitable porous medium model (i.e. Darcy, Forchheimer and/or Brinkman). Specific applications of flow in a porous-fluid layer system include flow of water under the Earth's surface (cf. Ewing 1997; Ewing \& Weekes 1998), flow of oil in underground reservoirs (cf. Allen 1984; Allen, Behie \& Trangenstein 1988), bioremediation of contaminated ground and related fields (cf. e.g. Suchomel, Chen \& Allen 1998; Chen et al. 1994), and manufacturing of composite materials used in the aircraft and automobile industries (cf. Blest et al. 1999a, b).

The present paper should not be confused with instability in a two-layer system composed of different immiscible fluids. This is another important problem, various aspects of which are studied in detail in Chen \& Crighton (1994), Hooper (1989), South \& Hooper (1999), Timoshin \& Hooper (2000), see also the references therein. In addition, the work here ought not to be confused with the resuspension problem wherein a two-layer situation is created by fluid flowing over a silty bottom which then creates a situation of a clean fluid layer overlying a layer of suspension. Work on the latter problem may be found in Schaflinger, Acrivos \& Zhang (1989), Zhang, Acrivos \& Schaflinger (1992), and Waller \& Schaflinger (1998).

In this paper we consider a fixed porous layer and our findings are significant. We discover a tri-modal structure to the neutral curves for instability. For $\hat{d} \leqslant 0.11$, instability is connected with the porous layer, whereas for $\hat{d} \approx 0.12$, the fluid layer takes over. This is seen by a bi-modal behaviour on one neutral curve. However, as $\hat{d}$ is increased, a second eigenvalue begins to dominate the instability picture and certainly by $\hat{d} \approx 0.15$, this eigenvalue is responsible for linear instability. Again, the fluid is now the dominant instability region. Here, we do not consider the limiting cases $\hat{d} \rightarrow 0$ or $\hat{d} \rightarrow \infty$. These cases could have important applications in such areas as mudslides, but will be investigated by a more careful asymptotic approach. We simply report on the tri-modal neutral curve behaviour and the associated changes of instability mechanism in the range $\hat{d}=0.1$ to $\hat{d}=0.3$.

\section{Governing equations}

We essentially employed the notation of Chen \& Chen (1988), adapted to the problem in hand. Thus, we consider a porous medium occupying the three-dimensional layer $\left\{x, y \in \mathfrak{R}^{2}\right\} \times\left\{z \in\left(-d_{m}, 0\right)\right\}$ with the fluid occupying the layer $\left\{x, y \in \mathfrak{R}^{2}\right\} \times$ $\{z \in(0, d)\}$. The interface between the porous medium and the fluid is at $z=0$. The governing equations in the fluid are the Navier-Stokes equations

$$
\begin{gathered}
\frac{\partial u_{i}}{\partial t}+u_{j} \frac{\partial u_{i}}{\partial x_{j}}=-\frac{1}{\rho} \frac{\partial p}{\partial x_{i}}+v \Delta u_{i}, \\
\frac{\partial u_{i}}{\partial x_{i}}=0,
\end{gathered}
$$

which are assumed to hold for time $t>0$. In these equations, $u_{i}$ and $p$ are velocity and pressure, and $\rho$ and $v$ are density and kinematic viscosity. Standard indicial notation is employed throughout, with $\Delta$ being the Laplace operator. In the porous medium, the governing equations are those of Darcy flow,

$$
\frac{1}{\chi} \frac{\partial u_{i}^{m}}{\partial t}=-\frac{1}{\rho} \frac{\partial p^{m}}{\partial x_{i}}-\frac{\nu}{K} u_{i}^{m},
$$




$$
\frac{\partial u_{i}^{m}}{\partial x_{i}}=0
$$

for $t>0$, where $u_{i}^{m}$ is the pore averaged velocity, $p^{m}$ the interstitial pressure, $\chi$ the porosity, $K$ the permeability, and superscript (or subscript) $m$ denotes porous medium where necessary.

To model the flow of a fluid over a porous medium, suitable conditions at the boundary between the two media are required. Deriving such conditions is proving problematic, see, for example, the approaches in Beavers \& Joseph (1967), Saffman (1971), Taylor (1971), Jones (1973), Caviglia, Morro \& Straughan (1992), Jäger \& Mikelic (1998), Ciesjko \& Kubik (1999), Jäger, Mikelic \& Neuss (1999), Murdoch \& Soliman (1999), Nield \& Bejan (1999) and McKay (2001). We here employ the experimentally suggested condition proposed by Beavers \& Joseph (1967), or that by Jones (1973). Even though the condition of Beavers \& Joseph (1967) precedes that of Jones (1973), it may be preferred as the latter is completely invariant. If we write $\boldsymbol{u}=\left(u_{1}, u_{2}, u_{3}\right)=(u, v, w)$ and $\boldsymbol{u}^{m}=\left(u_{1}^{m}, u_{2}^{m}, u_{3}^{m}\right)=\left(u^{m}, v^{m}, w^{m}\right)$ the Jones and Beavers-Joseph interface condition may be expressed as

$$
\frac{\partial u_{i}}{\partial z}+J \frac{\partial w}{\partial x_{i}}=\frac{\alpha}{\sqrt{K}}\left(u_{i}-u_{i}^{m}\right) \quad(i=1,2) .
$$

In (2.5), $J=1$ yields the Jones (1973) condition whereas that of Beavers \& Joseph (1967) follows by taking $J=0$, and $\alpha$ is the so-called Beavers-Joseph constant which must be determined by experiment and varies for different fluids and porous media. Straughan (2002) contains results for thermal convection for various values of $\alpha$ appropriate to real materials.

In addition to (2.5), we must assume that the normal velocity and the pressure are continuous across $z=0$, i.e. $w=w^{m}$ and $p^{m}=p$ (Beavers \& Joseph 1967). We observe that Payne \& Straughan (1998) show continuous dependence on the parameter $\alpha$ in the Jones condition when Darcy's law holds in the porous medium for Stokes' flow in the fluid layer. It is worth observing that the Beavers-Joseph condition has been successfully employed in the slow flow of a fluid past a porous sphere by Qin \& Kaloni (1993). Straughan (2004) concludes that if a linearized instability analysis is employed, then use of condition (2.5) with $J=0$ or $J=1$ is probably justified. Numerical computations for coupled fluid flow and porous flow problems are given by Discacciati, Miglio \& Quarteroni (2002) and Miglio, Quarteroni \& Saleri (2003). Das, Nassehi \& Wakeman (2002) is another numerical contribution employing a finitevolume method in three-dimensions where the porous medium may be anisotropic. They show that the direction of flow may reverse at the interface between the porous medium and fluid, a phenomenon also encountered here. Existence of a weak solution for Darcy porous media flow coupled to the Stokes equations in a fluid with the Beavers-Joseph interface boundary condition is proved in Layton, Schieweck \& Yotov (2003). Despite the concern in the literature over the correct interface boundary conditions, those of Jones or Beavers-Joseph have yielded good results when compared with experiment. Thus, we employ these conditions here. We find virtually no difference in the instability results whichever of the two boundary conditions is used. This agrees with what is found in thermal convection (cf. Straughan 2002, 2004).

\subsection{The basic flow}

For Poiseuille flow we assume a constant pressure gradient in the $x$-direction. The basic solution to equations (2.1) and (2.3) with the boundary conditions outlined 
above, together with $\boldsymbol{u}=\mathbf{0}$ on $z=d$ and $w^{m}=0$ on $z=-d_{m}$ is

$$
\left.\begin{array}{rl}
\bar{u}(z) & =\frac{1}{2} A_{1} z^{2}+A_{2} z+A_{3}, \quad \bar{v}=\bar{w}=0,0 \leqslant z \leqslant d, \\
\bar{u}^{m} & =-A_{1} K, \quad \bar{v}^{m}=\bar{w}^{m}=0, \quad-d_{m} \leqslant z \leqslant 0 .
\end{array}\right\}
$$

Note, we denote the base solution with an overbar. In (2.6), where

$$
\left.\begin{array}{l}
A_{1}=\frac{1}{\mu} \frac{\mathrm{d} p}{\mathrm{~d} x}, \quad A_{2}=\alpha A_{1} \sqrt{K}-\frac{\alpha A_{1} d^{2}+2 \alpha^{2} A_{1} d \sqrt{K}}{2(\alpha d+\sqrt{K})}, \\
A_{3}=-\frac{A_{1} d^{2} \sqrt{K}+2 \alpha A_{1} K d}{2(\alpha d+\sqrt{K})} .
\end{array}\right\}
$$

To interpret the relative magnitudes of velocity in both the fluid and porous layers, the length scale in the fluid layer is normalized by dividing by $d$ and that of the porous layer by $d_{m}$, and the dimensional basic velocities in both layers are normalized by dividing by $V$, the maximum of $\bar{u}(z)$. Then we have, respectively, the velocity function

$$
U(z)=\frac{\bar{u}(z)}{V}=\frac{z^{2}+2 B_{1} z+2 B_{2}}{2 B_{2}-B_{1}^{2}} \quad(z \in(0,1))
$$

for the fluid layer, and

$$
U_{m}=\frac{\bar{u}^{m}}{V}=\frac{2 \delta^{2}}{\left(B_{1}^{2}-2 B_{2}\right) \hat{d}^{2}} \quad(z \in(-1,0))
$$

for the porous layer, where

$$
B_{1}=-\frac{\alpha \hat{d}+2 \alpha^{2} \delta}{2(\alpha \hat{d}+\delta)}+\frac{\alpha \delta}{\hat{d}}, \quad B_{2}=-\frac{\delta \hat{d}+2 \alpha \delta^{2}}{2\left(\alpha \hat{d}^{2}+\delta \hat{d}\right)} .
$$

and $\delta$ is the Darcy number defined by $\delta=\sqrt{K} / d_{m}$. The resulting basic velocity profiles for three different $\hat{d}$ are displayed in figure 1 . Note that the presence of porous medium below breaks the symmetry of the basic flow in the fluid layer, so that the symmetry of classic Poiseuille flow precluding the existence of the odd shear mode may change, and in fact, as will be shown by the present analysis, the presence of both the porous medium and the shear in the fluid layer play crucial roles in the system stability.

\subsection{Perturbation equations}

We now derive perturbation equations and non-dimensionalize with scales of length, velocity, time and pressure in the fluid as $d, V, d / V, \mu V / d$, while in the porous layer they are $d_{m}, V_{m}, d_{m} / V_{m}, \mu V_{m} / d_{m}$, where $V_{m}=\bar{u}^{m}$. The linearized perturbation equations take the form

$$
\begin{gathered}
\frac{\partial u_{i}}{\partial x_{i}}=0 \\
\operatorname{Re}\left(\frac{\partial u_{i}}{\partial t}+u_{j} \frac{\partial \bar{u}_{i}}{\partial x_{j}}+\bar{u}_{j} \frac{\partial u_{i}}{\partial x_{j}}\right)=-\frac{\partial p}{\partial x_{i}}+\Delta u_{i}
\end{gathered}
$$

in $\mathfrak{R}^{2} \times(0,1) \times(0, \infty)$ and

$$
\begin{gathered}
\frac{\partial u^{m}}{\partial x_{i}^{m}}=0 \\
\frac{R e^{m}}{\chi} \frac{\partial u_{i}^{m}}{\partial t^{m}}+\frac{1}{\delta^{2}} u_{i}^{m}=-\frac{\partial p^{m}}{\partial x_{i}^{m}}
\end{gathered}
$$




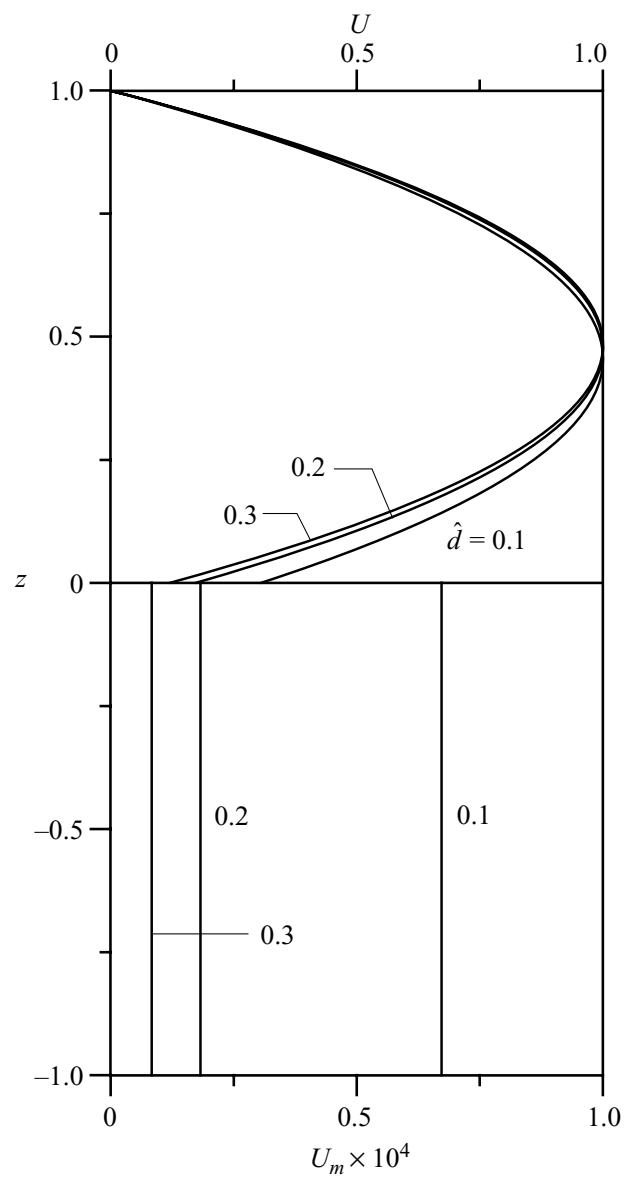

FIGURE 1. The basic states of dimensionless velocity in both fluid and porous layers for three assigned values of depth ratio. The maximum velocity in the fluid layer is set to 1 and the bottom scale show the relative magnitude of velocity in porous layer. The other parameters are $\delta=10^{-3}, \chi=0.3, \alpha=0.1$ and $J=0$.

in $\mathfrak{R}^{2} \times(-1,0) \times(0, \infty)$, where $R e$ and $R e^{m}$ are the Reynolds numbers in the fluid and porous layers given by $R e=V d / v$ and $R e^{m}=V_{m} d_{m} / \nu$. Note that

$$
R e^{m}=\frac{2 \delta^{2}}{\hat{d}^{3}\left(B_{1}^{2}-2 B_{2}\right)} R e .
$$

Normal modes are employed

$$
\begin{gathered}
u_{i}=u_{i}(z) \exp (\mathrm{i}(a x+b y-a c t)), \quad p=\pi(z) \exp (\mathrm{i}(a x+b y-a c t)), \quad(2.14 a, b) \\
u_{i}^{m}=u_{i}^{m}\left(z_{m}\right) \exp \left(\mathrm{i}\left(a_{m} x_{m}+b_{m} y_{m}-a_{m} c_{m} t_{m}\right)\right), p^{m}=\pi^{m}\left(z_{m}\right) \exp \left(\mathrm{i}\left(a_{m} x_{m}+b_{m} y_{m}-a_{m} c_{m} t_{m}\right)\right),
\end{gathered}
$$

to reduce the above equations to the form

$$
\mathrm{i} a u+\mathrm{i} b v+\mathrm{D} w=0,
$$




$$
\begin{gathered}
\operatorname{Re}(U-c) \mathrm{i} a u+\operatorname{Re}^{\prime} w=-\mathrm{i} a \pi+\left[\mathrm{D}^{2}-\left(a^{2}+b^{2}\right)\right] u, \\
\operatorname{Re}(U-c) \mathrm{i} a v=-\mathrm{i} b \pi+\left[\mathrm{D}^{2}-\left(a^{2}+b^{2}\right)\right] v, \\
\operatorname{Re}(U-c) \mathrm{i} a w=-\mathrm{D} \pi+\left[\mathrm{D}^{2}-\left(a^{2}+b^{2}\right)\right] w,
\end{gathered}
$$

and

$$
\begin{gathered}
\mathrm{i} a_{m} u_{m}+\mathrm{i} b_{m} v_{m}+\mathrm{D}_{p} w_{m}=0, \\
\left(\frac{1}{\delta^{2}}-R e^{m} \frac{\mathrm{i} a_{m} c_{m}}{\chi}\right) u_{m}+\mathrm{i} a_{m} \pi_{m}=0, \\
\left(\frac{1}{\delta^{2}}-R e^{m} \frac{\mathrm{i} a_{m} c_{m}}{\chi}\right) v_{m}+\mathrm{i} b_{m} \pi_{m}=0, \\
\left(\frac{1}{\delta^{2}}-\operatorname{Re}^{m} \frac{\mathrm{i} a_{m} c_{m}}{\chi}\right) w_{m}+\mathrm{D}_{P} \pi_{m}=0,
\end{gathered}
$$

where $\mathrm{D}=\mathrm{d} / \mathrm{d} z, U^{\prime}=\mathrm{d} U / \mathrm{d} z$, and $\mathrm{D}_{p}=\mathrm{d} / \mathrm{d} z_{m}$. Presumably the instability is dominated by two-dimensional disturbances; we employ Squire's theorem to reduce the above three-dimensional equations into an equivalent two-dimensional counterpart. The details of this procedure are standard and can be found in Drazin \& Reid (1981). The resultant two-dimensional equations are

$$
\begin{gathered}
\mathrm{i} a u+\mathrm{D} w=0, \\
\operatorname{Re}(U-c) \mathrm{i} a u+\operatorname{Re}^{\prime} w=-\mathrm{i} a \pi+\left(\mathrm{D}^{2}-a^{2}\right) u, \\
\operatorname{Re}(U-c) \mathrm{i} a w=-\mathrm{D} \pi+\left(\mathrm{D}^{2}-a^{2}\right) w,
\end{gathered}
$$

for $z \in(0,1)$, and

$$
\begin{gathered}
\mathrm{i} a_{m} u_{m}+\mathrm{D}_{p} w_{m}=0, \\
\left(\frac{1}{\delta^{2}}-R e^{m} \frac{\mathrm{i} a_{m} c_{m}}{\chi}\right) u_{m}+\mathrm{i} a_{m} \pi_{m}=0, \\
\left(\frac{1}{\delta^{2}}-R e^{m} \frac{\mathrm{i} a_{m} c_{m}}{\chi}\right) w_{m}+\mathrm{D}_{P} \pi_{m}=0,
\end{gathered}
$$

for $z \in(-1,0)$.

We next introduce the streamfunction $\psi$ by $u=\partial \psi / \partial z, w=-\partial \psi / \partial x$, with a similar definition for $\psi_{m}$ and further introduce eigenfunctions $\phi$ and $\phi^{m}$ by

$$
\psi=\phi(z) \exp (\mathrm{i} a(x-c t)), \quad \psi^{m}=\phi^{m}\left(z_{m}\right) \exp \left(\mathrm{i} a_{m}\left(x_{m}-c_{m} t_{m}\right)\right) .
$$

The differential equations (2.17) and (2.18) may now be reduced to the following two equations,

$$
\left(\mathrm{D}^{2}-a^{2}\right)^{2} \phi=\mathrm{i} a \operatorname{Re}(U-c)\left(\mathrm{D}^{2}-a^{2}\right) \phi-\mathrm{i} a \operatorname{Re} U^{\prime \prime} \phi, \quad z \in(0,1),
$$




$$
\left(\frac{1}{\delta^{2}}-R e^{m} \frac{\mathrm{i} a_{m} c_{m}}{\chi}\right)\left(\mathrm{D}_{p}^{2}-a_{m}^{2}\right) \phi_{m}=0, \quad z_{m} \in(-1,0) .
$$

It is worth recording the following relations between $a, a_{m}, c$ and $c_{m}$,

$$
a=\hat{d} a_{m}, \quad c R e=c_{m} \hat{d} R e^{m} .
$$

Equations (2.20) and (2.21) comprise a sixth-order system. To this we append the boundary conditions, on the upper plane $z=1$,

$$
\phi=\mathrm{D} \phi=0,
$$

while on the base of the porous layer $z_{m}=-1$,

$$
\phi^{m}=0 \text {. }
$$

On the interface $z_{m}=z=0$, we have

$$
\begin{gathered}
\operatorname{Re} \phi=R e^{m} \phi^{m}, \\
\mathrm{D}^{2} \phi+J a^{2} \phi-\frac{\alpha \hat{d}}{\delta} \mathrm{D} \phi+\frac{\alpha \hat{d}^{2} R e^{m}}{\delta R e} \mathrm{D}_{p} \phi^{m}=0, \quad z=0,
\end{gathered}
$$

and also on $z=0$,

$$
\left(\operatorname{Re}^{m} \frac{\mathrm{i} a_{m} c_{m}}{\chi}-\frac{1}{\delta^{2}}\right) \mathrm{D}_{p} \phi^{m}=\frac{R e}{\hat{d}^{3} R e^{m}}\left[\left(\mathrm{D}^{2}-a^{2}\right) \mathrm{D} \phi-\mathrm{i} a \operatorname{Re}(U-c) \mathrm{D} \phi+\mathrm{i} a \operatorname{Re} U^{\prime} \phi\right] .
$$

Thus, the complete system to be solved is comprised of equations (2.20) and (2.21) together with the six boundary conditions (2.22)-(2.27), which is an eigenvalue problem.

\section{Numerical results and discussion}

We solve the above eigenvalue problem by means of a $\mathrm{D}^{2}$ Chebyshev tau method (cf. Dongarra, Straughan \& Walker (1996)). To do this we rewrite (2.20) as two second-order equations

$$
\begin{gathered}
\left(\mathrm{D}^{2}-a^{2}\right) \phi-\xi=0, \\
\left(\mathrm{D}^{2}-a^{2}\right) \xi=\mathrm{i} a \operatorname{Re}(U-c) \xi-\mathrm{i} a \operatorname{Re} U^{\prime \prime} \phi .
\end{gathered}
$$

We then solve (2.21), (3.1), (3.2) for $\phi, \xi, \phi^{m}$ (regarded as independent variables) in the Chebyshev domain $(-1,1)$. Details of the numerical procedure are similar to those in Straughan (2001, 2002).

The numerical results presented here are very accurate and to ensure this we have checked convergence. By varying the number of polynomials, $N$, it is found that 50 polynomials usually yields 8 decimal places of accuracy in 64 bit arithmetic, for the $R e$ values we are working with. For example, at $a=1, R e=10^{4}$ with $\hat{d}=0.1, \delta=0.001$, $\chi=0.3, \alpha=0.1$ and $J=0$, the leading eigenvalue $c_{r}+\mathrm{i} c_{i}$ for $N=50,60$ and 70 is always $0.47942476+\mathrm{i} 0.03274867$. The results we report are obtained with $N=50$. In the following analyses, we fix major parameters at representative values of $\hat{d}=0.13$, $\delta=10^{-3}, \chi=0.3, \alpha=0.1$ and $J=0$ to study the variation of stability with $\hat{d}, \alpha$ and $\delta$. As the effect of one parameter is studied, the other parameters are fixed as shown above. Some practical properties of many materials of porous media can be found in Straughan (2002 table 1). 


\begin{tabular}{|c|c|c|c|}
\hline$a$ & $R e$ & $c_{r}$ & $c_{i}$ \\
\hline \multirow[t]{8}{*}{1.45} & \multirow[t]{2}{*}{9650} & 0.277278 & $-0.175791 \times 10^{-2}$ \\
\hline & & 0.421976 & $-0.184145 \times 10^{-2}$ \\
\hline & \multirow[t]{2}{*}{9700} & 0.421619 & $-0.149724 \times 10^{-2}$ \\
\hline & & 0.277073 & $-0.169746 \times 10^{-2}$ \\
\hline & \multirow[t]{2}{*}{9900} & 0.420221 & $-0.136822 \times 10^{-3}$ \\
\hline & & 0.276262 & $-0.146499 \times 10^{-2}$ \\
\hline & \multirow[t]{2}{*}{9950} & 0.419879 & $+0.199238 \times 10^{-3}$ \\
\hline & & 0.276061 & $-0.140912 \times 10^{-2}$ \\
\hline \multirow[t]{8}{*}{1.65} & \multirow[t]{2}{*}{7150} & 0.303031 & $-0.469375 \times 10^{-4}$ \\
\hline & & 0.429535 & $-0.224921 \times 10^{-1}$ \\
\hline & \multirow[t]{2}{*}{7200} & 0.302767 & $+0.535402 \times 10^{-4}$ \\
\hline & & 0.428949 & $-0.220648 \times 10^{-1}$ \\
\hline & \multirow[t]{2}{*}{10600} & 0.288131 & $+0.293034 \times 10^{-2}$ \\
\hline & & 0.398393 & $+0.289542 \times 10^{-2}$ \\
\hline & \multirow[t]{2}{*}{10650} & 0.398055 & $+0.321401 \times 10^{-2}$ \\
\hline & & 0.287947 & $+0.293139 \times 10^{-2}$ \\
\hline \multirow[t]{8}{*}{1.95} & \multirow[t]{2}{*}{9400} & 0.314078 & $-0.282455 \times 10^{-2}$ \\
\hline & & 0.384153 & $-0.295594 \times 10^{-2}$ \\
\hline & \multirow[t]{2}{*}{9450} & 0.383708 & $-0.253630 \times 10^{-2}$ \\
\hline & & 0.313867 & $-0.293265 \times 10^{-2}$ \\
\hline & \multirow[t]{2}{*}{9750} & 0.381136 & $-0.287064 \times 10^{-3}$ \\
\hline & & 0.312590 & $-0.361514 \times 10^{-2}$ \\
\hline & \multirow[t]{2}{*}{9800} & 0.380725 & $+0.387157 \times 10^{-3}$ \\
\hline & & 0.312374 & $-0.373397 \times 10^{-2}$ \\
\hline
\end{tabular}

TABLE 1. The variation of the first two eigenvalues with largest imaginary parts, with respect to $R e$, for several assigned values of $a ; \hat{d}=0.13$.

\subsection{Depth ratio $\hat{d}$ effects}

The depth ratio $\hat{d}$ is the major parameter influencing the stability of the superposed fluid-porous layer system (Chen \& Chen 1988). Figure 2 represents the neutral curves $\left(c_{i}=0\right)$ for various values of $\hat{d}$. In figures $2(a)$ and $2(b)$, the neutral curves display a bi-modal structure. In figure $2(a)$, the dominant mode of instability is found for $\hat{d}=0.11, a_{c}=0.84$ with $R e^{c}=7234$, the subscript (or superscript) $c$ denoting critical value where necessary. The corresponding eigenfunction in figure $3(a)$ shows the strong effect the porous medium has, and indeed, a flow reversal near the interface reveals the involvement of the porous medium in the stability owing to the space restriction within the shallow fluid layer. When $\hat{d}=0.12$ (figure $2 b$ ), the dominant mode of instability is found at $a_{c}=2.46, R e^{c}=7560$. The corresponding eigenfunction (figure $3 b$ ) shows strong movement in the fluid, but with little effect now in the porous medium. Flow reversal is still evident in the upper part of the fluid layer while it disappears completely in the porous layer, and the real part of $\phi$ is approximately antisymmetric with respect to the centreline of the fluid layer (or an odd function). In figure $2(c), \hat{d}=0.121$, the dominant mode is still in the fluid $\left(a_{c} \approx 2.5\right)$, but now a second eigenvalue begins to enter the picture (the v-shaped protrusion). The broken curves in figures $2(a)-2(f)$ represent the real part of $c$ and in figures $2(c)-2(f)$ the break in this curve is due to mode switching (i.e. the second eigenvalue entering the picture, which will be explained in more detail in table 1 and figure 4).

In figure $2(d)$, the second eigenvalue now represents the dominant mode of instability, as indicated by the lowest minimum at the middle. The eigenfunction, 

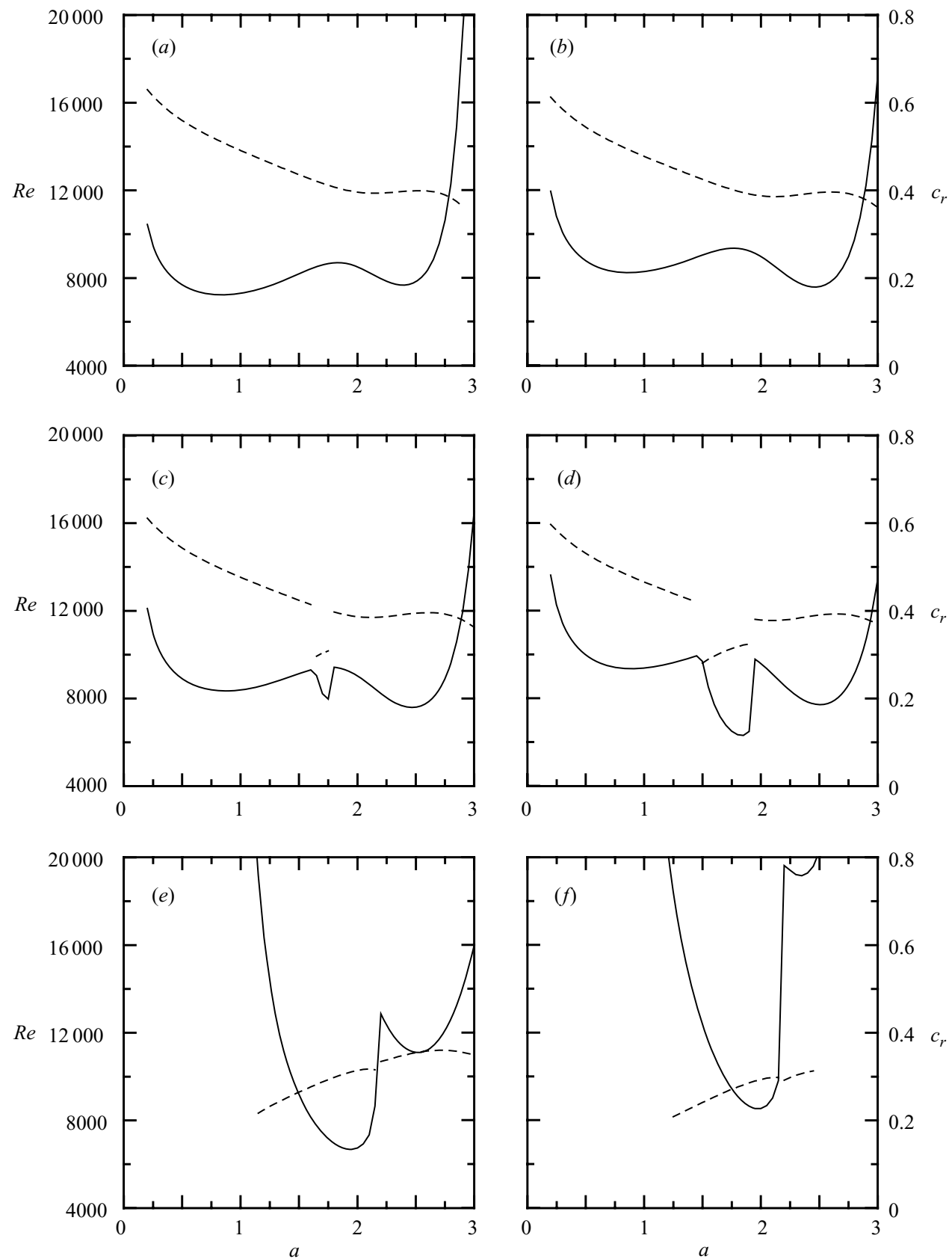

FIGURE 2. The variations of the neutral curve indicated by the solid line and corresponding oscillatory frequency by the dashed line with several assigned values of depth ratio; $(a)$ $\hat{d}=0.11,(b) \hat{d}=0.12,(c) \hat{d}=0.121,(d) \hat{d}=0.13$, (e) $\hat{d}=0.2,(f) \hat{d}=0.3$. The other parameters are $\delta=10^{-3}, \chi=0.3, \alpha=0.1$ and $J=0$.

figure 3(c), shows that the instability is now largely controlled by the fluid layer and the real part of $\phi$ is approximately symmetric with respect to the centreline of the fluid layer (an even function). Note that, in the tri-modal neutral curve such as that of figure $2(d)$, the eigenfunction corresponding to the local minimum at the 

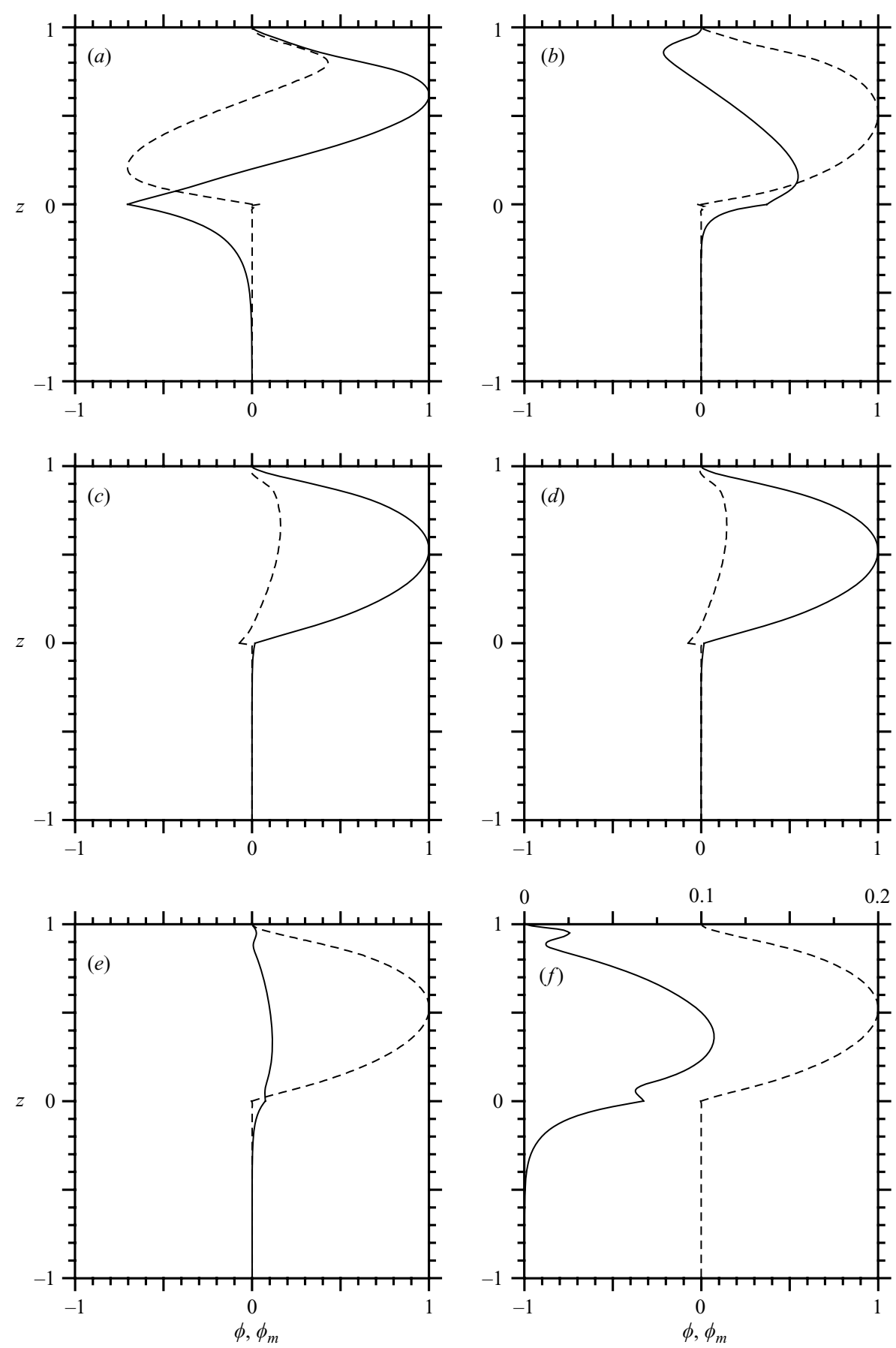

FIGURE 3. The variations of normalized dimensional streamfunction amplitude with respect to several assigned values of the depth ratio at a critical state, the solid lines represent the real parts and the dashed lines represent the imaginary parts; $(a) \hat{d}=0.11, a_{c}=0.84, R e^{c}=7234$; (b) $\hat{d}=0.12, a_{c}=2.46, R e^{c}=7584 ;(c) \hat{d}=0.13, a_{c}=1.84, R e^{c}=6297 ;(d) \hat{d}=0.15, a_{c}=1.89$, $R e^{c}=5961 ;(e) \hat{d}=0.16, a_{c}=1.91, R e^{c}=6032 ;(f) \hat{d}=0.2, a_{c}=1.94, R e^{c}=6676$. Note that in $(f)$ the real part is magnified with respect to the scale of the above horizontal axis. The other parameters are $\delta=10^{-3}, \chi=0.3, \alpha=0.1$ and $J=0$. 
left is similar to that of figure $3(a)$, and that to the local minimum at the right is similar to that of figure $3(b)$. As $\hat{d}$ is increased to 0.2 , see figure $2(e)$, the lefthand lobe of the neutral curve corresponding to the porous-layer dominance has disappeared and the second eigenvalue is clearly dominant. In figure $2(f), \hat{d}=0.3$, the right-hand lobe corresponding to the fluid-layer dominance has almost disappeared from the scene with the second eigenvalue dominating everything. Figures $3(d)-3(f)$ show the eigenfunctions for $\hat{d}=0.15,0.16,0.2$, respectively, suggesting that the second eigenvalue accounts for an instability mode largely confined to the fluid layer.

According to Chen \& Chen (1988), who investigated the onset of thermal convection in the superposed fluid-porous layers heated from below, the mode corresponding to the left-hand lobe of the neutral curve (figure $2 c$ ) is the so-called porous-layer mode (Worster 1992) that the onset of instability occurs in both the fluid and porous layers while the porous medium largely controls the stability. The mode corresponding to the right-hand lobe is the fluid-layer mode (Worster 1992) so that the onset of instability is largely confined to the fluid layer and the porous layer plays virtually no role in stability. The switch between the fluid-layer and the porous-layer modes depends primarily on the relative depth of the fluid layer, or $\hat{d}$. For a large $\hat{d}$ the fluid-layer dominates and for a small $\hat{d}$ the porous-layer dominates.

The third mode corresponding to the middle lobe was not present in the Bénard problem of Chen \& Chen (1988). In the Bénard problem, the basic flow is quiescent so there is no shear in the horizontal direction, leading to the porous-layer mode shifting dramatically into the fluid-layer mode at $\hat{d} \approx 0.13$, i.e. no part in the transition played by the third mode. It is therefore implied that the third mode results from the shear in the fluid layer. To confirm this scenario, we observe the eigenfunctions of figure 3 as follows. As noted previously, by observing figure $3(b)$ regarding the eigenfunction of this fluid-layer mode, it is found that the $\phi$ is approximately an odd-symmetry function with respect to the centreline of the fluid layer. Accordingly, we will call this mode the odd-fluid-layer mode in order to differentiate from the third mode whose $\phi$ (see figure $3 c$ ) is virtually an even-symmetry function in the fluid layer. We recall that in the single-layer plane Poiseuille flow, the symmetry of the basic flow precludes the possibility that odd disturbances can be unstable, only the even-shear-mode dominates. As a consequence, we imply that the third mode is obviously the even-shear-mode of the Poiseuille flow so that it will be called the even-fluid-layer mode afterwards.

We also note that the present stability characteristics are very different from those revealed by Tilley et al. $(1994 a, b)$ who investigated the stability of the plane Poiseuille flow of two immiscible oil-water superposed fluids, although they also found tri-modal instability in their system. In addition to the even-shear-mode, they found that the interfacial mode (triggered by the shear at interface) and the odd shear mode (triggered by the breaking symmetry of the basic flow) can become the primary instability depending mainly on the relative channel thickness, and the interfacial mode becomes the dominant instability when the depth ratio ( $\hat{d}$, oil-to-water) is large. The interfacial mode is obviously a result of the presence of the immiscible interface. In the present system, before the third mode (or the even-fluid-layer mode) is initiated, the stability is bi-modal, competing by the odd-fluid-layer and porous-layer modes due to changing $\hat{d}$. After the even-fluid-layer-mode is initiated, the fluid-layer dominance becomes more significant as $\hat{d}$ increases and, eventually, both the porous-layer and the odd-fluid-layer modes are completely suppressed. Under such a circumstance, the presence of the porous layer can be virtually ignored and the whole system behaves like a plane Poiseuille flow with a porous-slip boundary below. 

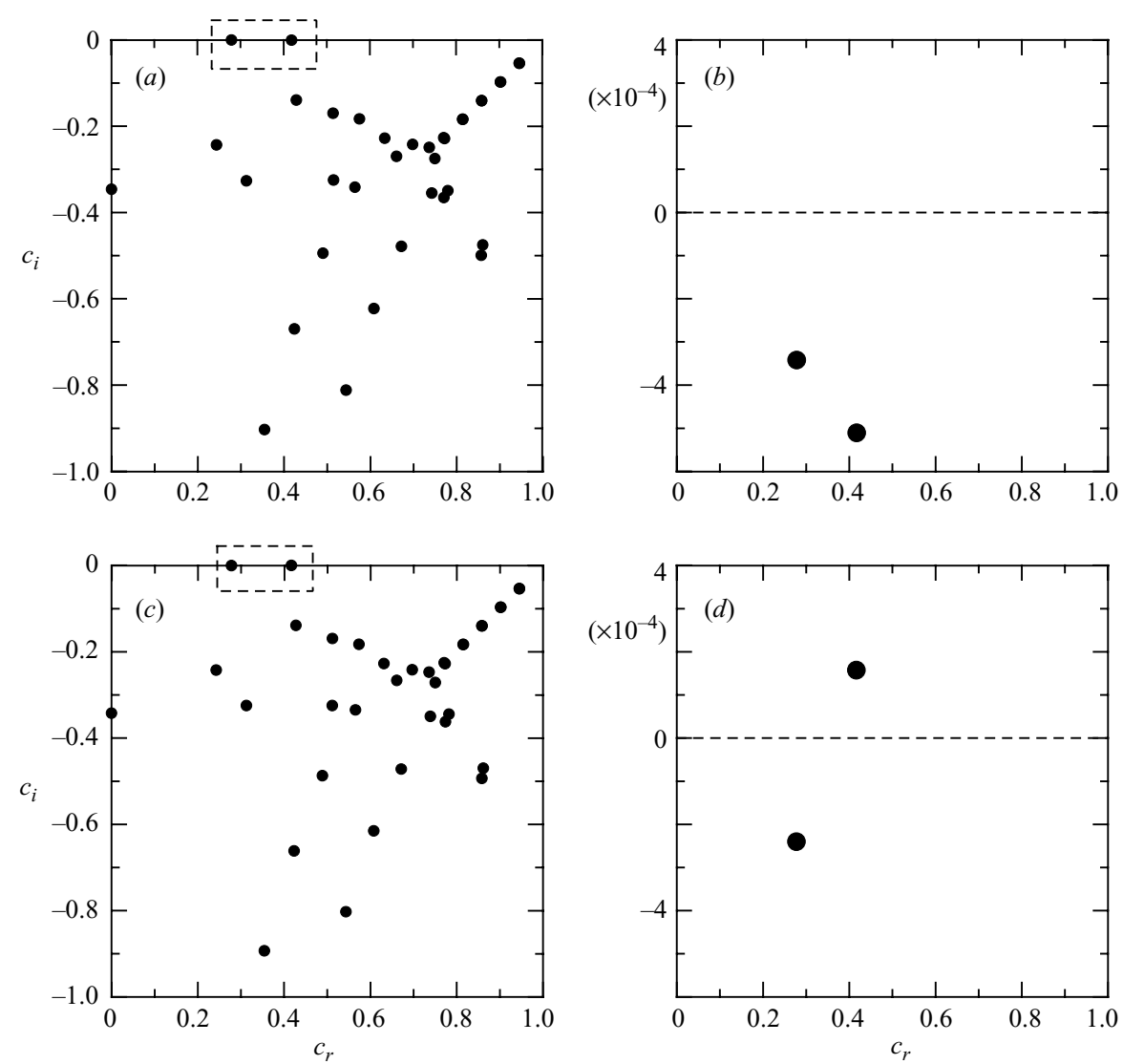

Figure 4. The spectrum of eigenvalue at $a=1.48$ with $\hat{d}=0.13$, (a) $R e=9900$, (c) $R e=10000$, $(b)$ and $(d)$ are the magnifications of the square region for the first two leading eigenvalues for (a) and $(c)$, respectively. The other parameters are $\delta=10^{-3}, \chi=0.3, \alpha=0.1$ and $J=0$.

The above discussion indicates that the mode switching from bi-modal stability to tri-modal stability plays a crucial role in the present system. We therefore show the detail of the mode switching in table 1 . Here, we fix $\hat{d}=0.13$ and vary $R e$ for fixed values of $a$. For example, it can be seen that with $a=1.45$ when $R e=9650$ the eigenvalue with smaller $c_{r}=0.277278$ is the leading one whereas when $R e=9700$ the next eigenvalue with larger $c_{r}=0.421619$ takes over to control the stability. Such eigenvalue switching is allowed in complex space and does not contradict continuity. At $a=1.65$, the change of leading eigenvalue also occurs between $R e=10600$ and 10650 , while the stability now is dominated by the mode with smaller $c_{r}$ in the lower range of $R e$ between 7150 and 7200 . At $a=1.95$, we observe another example of the change of the first two leading eigenvalues with $R e$.

The mode switching can also be seen from the eigenvalue spectrum for $\hat{d}=0.13$, $a=1.48$ shown in figure 4. In the upper part of figures $4(a)$ and 4(c), we can see clearly that two eigenvalues are dominant. As $R e$ goes from 9900 to $10^{4}$ the leading eigenvalues change places, i.e. the mode switching occurs, see the blown-up detail in figures $4(b)$ and $4(d)$. For $R e=9900$, the left-hand mode with smaller $c_{r}$ is closer to the neutral state $\left(c_{i}=0\right.$, figure $\left.4 b\right)$. For $R e=10000$, the value of $c_{i}$ for the right-hand 


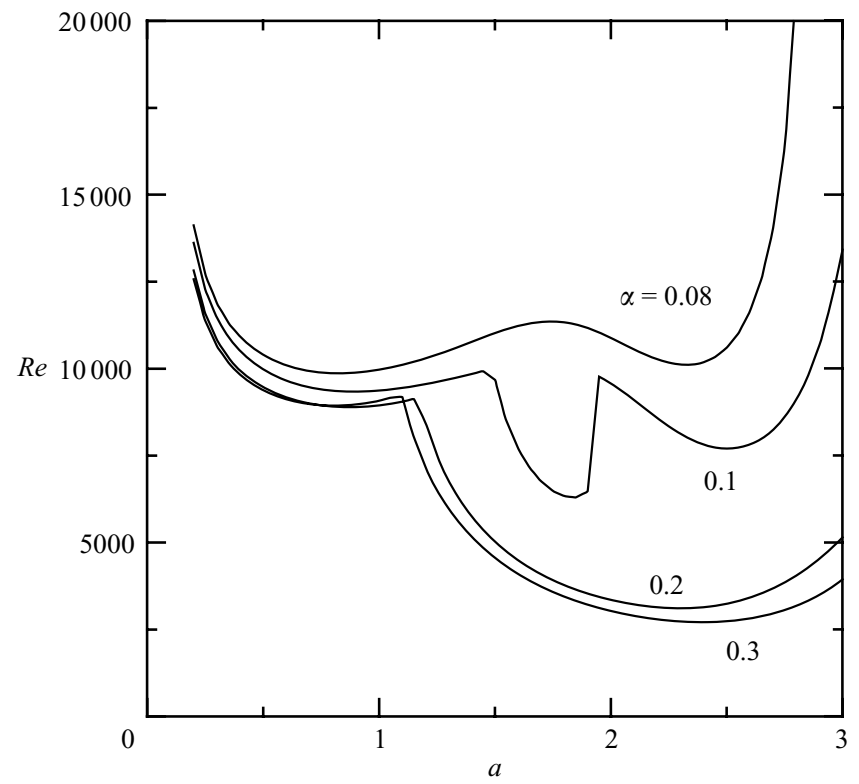

FIGURE 5. The variations of neutral curves with several assigned values of Beavers-Joseph constant $\alpha$. The other parameters are $\delta=10^{-3}, \chi=0.3, \hat{d}=0.13$ and $J=0$.

mode increases faster than the left-hand one (figure $4 d$ ), and becomes positive to dominate the stability of the system.

\subsection{The Beavers-Joseph constant $\alpha$ effects}

Examining effects of $\alpha$ can help to clarify the physical mechanism triggering the trimodal instability. We compute the neutral curves for four different $\alpha$ and the results are shown in figure 5. According to (2.5), the Beavers-Joseph interface condition, the value of $\alpha$ can be seen as being proportional to the magnitude of the velocity gradient above the interface, i.e. a larger $\alpha$ corresponds to a larger velocity gradient or a larger shear stress above the interface. Accordingly, for $\alpha=0.08$, the shear stress above the interface is small and the instability occurs in both the fluid and porous layers and the bi-modal stability prevails (similar to figures $2 a$ and $2 b$ ). For $\alpha=0.1$, the shear stress increases and triggers the even-fluid-layer mode, resulting in the tri-modal stability. For $\alpha=0.2$ and 0.3 , the shear stress at the interface is so large that the even-fluid-layer mode instability is enhanced and dominates the system completely.

In view of the stability criteria, results of figure 5 conclude that a larger $\alpha(\geqslant 0.2)$ can more easily trigger the instability in the fluid layer, leading to a more unstable system. On the other hand, a smaller $\alpha(\leqslant 0.08)$ leads to an instability mode in both layers, so that the resistance in a porous medium makes the system more stable. A moderate value of $\alpha(\approx 0.1)$ gives rise to the even-fluid-layer-mode instability, which serves to terminate the bi-modal instability so that the system is transferred to the fluid-layer-dominant state by way of a tri-modal instability.

\subsection{The Darcy number $\delta$ effect}

In figure 6 , six neutral curves for different $\delta$ are presented. Note that the value of $\delta$ equivalently accounts for the permeability of the porous layer. The three curves for $\delta \geqslant 2 \times 10^{-3}$ show that the critical mode has a small $a$, being similar to the case of figure 2(a) that the porous-layer mode prevails. For these cases, the porous 


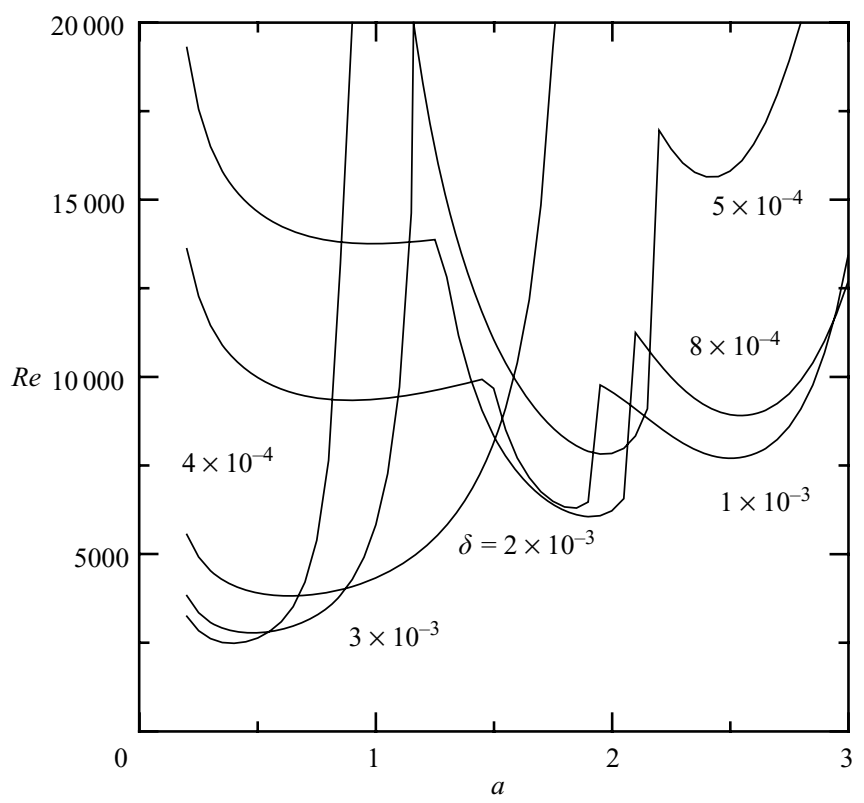

FIGURE 6. The variations of neutral curves with several assigned values of Darcy number $\delta$. The other parameters are $\alpha=0.1, \chi=0.3, \hat{d}=0.13$ and $J=0$.

layer dominates the system because, owing to a larger $\delta$ (or a larger permeability in the porous layer), the instability can more easily occur in the porous medium. Consequently, the system becomes more unstable for a larger $\delta$. As $\delta$ is decreased to $1 \times 10^{-3}$, owing to the smaller permeability (or the higher resistance) in the porous layer, more fluid is forced to move within the fluid layer so that the velocity gradient (or the shear) above the interface increases. As a result, the even-fluid-layer mode is triggered and the fluid layer becomes dominant in the system. As $\delta$ is decreased further, the fluid-layer dominance is enhanced and eventually the fluid layer controls the stability completely. For such a system, the porous layer has a small permeability (or equivalently a small porosity) so that the presence of the porous layer plays no role in the stability of the system. Namely, the whole system behaves like a plane Poiseuille flow with a porous-slip boundary below.

\section{Conclusions}

We have studied the linear instability problem for the situation of Poiseuille flow when the configuration is one of a Newtonian fluid overlying a porous layer saturated with the same fluid. The linearized analysis has revealed an instability scenario where three distinct regimes of instability are found as physical parameters $\hat{d}, \alpha$ and $\delta$ change. Based on the parameter values considered in the present study, the critical values are $\hat{d} \approx 0.13$ or $\alpha \approx 0.1$ or $\delta \approx 10^{-3}$ at which the stability is shifted from bi-modal to tri-modal. Generally, for a smaller $\hat{d}$, a smaller $\alpha$, or a larger $\delta$, the bi-modal instability prevails (the porous-layer and the odd-fluid-layer modes exist simultaneously) and the porous layer predominates the system. At about these critical values, the instability is tri-modal, the even-fluid-layer mode (or the third mode) instability is initiated to compete with the other two modes. As $\hat{d}$ or $\alpha$ increases or as $\delta$ decreases, the even-fluid-layer mode instability is enhanced, leading to the fluid layer controlling the 
stability completely. The even-fluid-layer mode is initiated by the shear in the fluid layer. As this mode prevails, the present system behaves like the plane Poiseuille flow with a solid boundary at the top and a porous-slip boundary below.

We define these three instability modes in a more precise way as follows. The porous layer mode corresponds to the local minimum of the left-hand lobe of the neutral curve (figure $2 a$ ). As it prevails, the porous layer dominates the stability of the system and a significant portion of perturbation of the flow occurs in the porous layer. The odd-fluid-layer mode corresponds to the local minimum of the right-hand lobe of the neutral curve (figure $2 b$ ). As it prevails, the fluid layer dominates the stability of the system and most of the perturbed flow occurs in the fluid layer, in which the perturbed streamfunction is an odd-symmetric function with respect to the centreline of the fluid layer. The even-fluid-layer mode corresponds to the local minimum of the central lobe of the neutral curve (figure $2 d$ ). As it prevails, the fluid layer dominates the stability of the system and the perturbed streamfunction is an even-symmetric function with respect to the centreline of the fluid layer. The porouslayer and odd-fluid-layer modes exist only in small ranges of the physical parameters considered. Beyond these parameter ranges, the even-fluid-layer mode prevails and dominates the stability of the system.

It is believed that these three instability modes are triggered by the shear in the fluid layer. Explanations are given in the following. For the porous-layer mode, the instability initiates in the fluid layer and penetrates into the porous layer through the fluid-porous layer interface; it can be seen from figure $3(a)$ that most of the perturbation in the porous layer is confined to the region close to the interface. For the odd-fluid-layer mode, the instability also initiates in the fluid layer but remains in the fluid layer owing to the large restriction to the fluid motion in the porous layer. However, it will be replaced by the even-fluid-layer mode when the porous layer effect becomes negligible and the system behaves like a plane Poiseuille flow with a solid boundary at the top and a slip-porous boundary at bottom, which occurs when the depth ratio is large, the Darcy number is small, or the Beavers-Joseph constant is large.

Since for a plane Poiseuille flow, the even-symmetry of the basic Poiseuille flow precludes the possibility of the odd-shear-mode instability. Accordingly, the oddfluid-layer exists only when the porous layer still plays a role, although the role may not be as significant as in the case where the porous-layer mode prevails. For the even-fluid-layer mode, the mechanism triggering the intability is also the shear in the fluid layer. This mode dominates the superposed-layer system in a large range of parameters, in which the porous layer behaves like a slip-porous boundary to the system and the whole system behaves like a plane Poiseuille flow with a slip-porous boundary below.

Based on the above discussions, we may imply that when the porosity of the porous layer approaches zero, only the even-fluid-layer mode persists because under such circumstances the whole system behaves like a plane Poiseuille flow so that the even-shear mode dominates.

The present stability characteristics are different from two other similar systems considered previously. To the Bénard problem considered in Chen \& Chen (1988) in which no shear exists in the basic flow, only the odd-fluid-layer and porous-layer modes compete to dominate the system. To the two immiscible superposed fluids considered in Tilley et al. $(1994 a, b)$ in which a sharp interface lies between two fluids, three modes exist simultaneously: the even-mode, the odd-mode, and the interfacial shear mode; and the interfacial shear mode becomes dominant when $\hat{d}$ is large. In 
the present system, both the bi-modal and the tri-modal instabilities exist only in small ranges of $\hat{d}, \alpha$ and $\delta$. For the cases beyond these ranges, the even-fluid-layer mode is enhanced to dominate the system so that the fluid layer controls the stability completely.

The authors thank the financial support for this research from National Science Council (Taiwan) through grants NSC 92-2212-E-002-007 and NSC 92-2212-E-132001.

\section{REFERENCES}

Allen, M. B. 1984 Collocation Techniques for Modeling Compositional Flows in Oil Reservoirs. Springer.

Allen, M. B., Behie, A. \& Trangenstein, J. A. 1988 Multiphase Flow in Porous Media: Mechanics, Mathematics, and Numerics. Springer.

Beavers, G. S. \& Joseph, D. D. 1967 Boundary conditions at a naturally permeable wall. J. Fluid Mech. 30, 197-207.

Blest, D. C., Duffy, B. R., McKee, S. \& Zulkifle, A. K. 1999 a Curing simulation of thermoset composites. Composites A 30, 1289-1309.

Blest, D. C., McKee, S., Zulkifle, A. K. \&. Marshall, P. $1999 b$ Curing simulation by autoclave resin infusion. Composites Sci. Technol. 59, 2297-2313.

Butler, K. M. \& FARrell, B. F. 1992 Three-dimensional optimal perturbations in viscous shear flow. Phys. Fluids A 4, 1637-1650.

Caviglia, G., Morro, A. \& Straughan, B. 1992 Reflection and refraction at a variable porosity interface. J. Acoust. Soc. Am. 92, 1113-1119.

Chen, B., Cunningham, A., Ewing, R., Peralta, R. \& Visser, E. 1994 Two-dimensional modeling of microscale transport and biotransformation in porous media. Numer. Meth. Partial Diff. Equations 10, 65-83.

Chen, F. \& Chen, C. F. 1988 Onset of finger convection in a horizontal porous layer underlying a fluid layer. J. Heat Transfer 110, 403-409.

Chen, K. P. \& Crighton, D. G. 1994 Instability of the large Reynolds number flow of a Newtonian fluid over a viscoelastic fluid. Phys. Fluids A 6, 152-163.

Ciesjko, M. \& KubiK, J. 1999 Compatibility conditions in the fluid-fluid saturated porous solid contact problems. Transport Porous Med. 34, 319-336.

Das, D. B., Nassehi, V. \& Wakeman, R. J. 2002 A finite volume model for the hydrodynamics of combined free and porous flow in sub-surface regions. Adv. Environ. Res. 7, 35-58.

Discacciati, M., Miglio, E. \& Quarteroni, A. 2002 Mathematical and numerical models for coupling surface and groundwater flows. Appl. Numer. Maths 43, 57-74.

Dongarra, J. J., Straughan, B. \& Walker, D. W. 1996 Chebyshev tau-QZ algorithm methods for calculating spectra of hydrodynamic stability problems. Appl. Numer. Maths 22, 399-435.

Drazin, P. G. \& ReID, W. H. 1981 Hydrodynamic Stability. Cambridge University Press.

EwING, R. E. 1997 The need for multidisciplinary involvement in groundwater contaminant simulations. Proceedings of Next Generation Environmental Models and Computational Methods (ed. G. Delic \& M. Wheeler), pp. 227-245. SIAM, Philadelphia, PA.

Ewing, R. E. \& WeEkes, S. 1998 Numerical methods for contaminant transport in porous media. Computational Maths 202, 75-95.

Hooper, A. P. 1989 The stability of two superposed viscous fluids in a channel. Phys. Fluids A 1, $1133-1142$.

JäGER, W. \& Mikelic, A. 1998 On the interface boundary condition by Beavers, Joseph and Saffman. Interdisziplinäres Zentrum für Wissenschaftliches Rechnen der Universität Heidelberg. Preprint 98-12.

JäGer, W., Mikelic, A. \& Neuss, N. 1999 Asymptotic analysis of the laminar viscous flow over a porous bed. Interdisziplinäres Zentrum für Wissenschaftliches Rechnen der Universität Heidelberg. Preprint 99-33.

Jones, I. P. 1973 Low Reynolds number flow past a porous spherical shell. Proc. Camb. Phil. Soc. 73, 231-238. 
Layton, W. J., Schieweck, F. \& Yotov, I. 2003 Coupling fluid flow with porous media flow. SIAM J. Numer. Anal. 40, 2195-2218.

McKaY, G. 2001 The Beavers and Joseph condition for velocity slip at the surface of a porous medium. In Continuum Mechanics and Applications in Geophysics and the Environment (ed. B. Straughan, R. Greve, H. Ehrentraut \& Y. Wang). Springer.

Miglio, E., Quarteroni, A. \& Saleri, F. 2003 Coupling of free surface and groundwater flows. Computers Fluids 32, 73-83.

Murdoch, A. J. \& Soliman, A. 1999 On the slip-boundary condition for liquid flow over planar porous boundaries. Proc. R. Soc. Lond. A 455, 1315-1340.

Nield, D. A. 1983 The boundary correction for the Rayleigh-Darcy problem; limitations of the Brinkman equation. J. Fluid Mech. 128, 37-46.

Nield, D. A. 1991 The limitations of the Brinkman-Forchheimer equation in modeling flow in a saturated porous mednum and at an interface. Intl J. Heat Fluid Flow 12, 269-272.

NIELD, D. A. 1998 Modelling the effect of surface tension on the onset of natural convection in a saturated porous medium. Transport Porous Med. 31, 365-368.

Nield, D. A. \& Bejan, A. 1999 Convection in Porous Media. Springer.

Payne, L. E. \& Straughan, B. 1998 Analysis of the boundary condition at the interface between a viscous fluid and a porous medium and related modeling questions. J. Maths Pures Appl. 77, 317-354.

QIn, Y. \& Kaloni, P. N. 1993 Creeping flow past a porous spherical shell. Z. Angew. Math. Mech. 73, 77-84.

Saffman, P. 1971 On the boundary conditions at the surface of a porous medium. Stud. Appl. Maths 50, 93-101.

Schaflinger, U., Acrivos, A. \& Zhang, K. 1989 Viscous resuspension of a sediment within a laminar and stratified flow. Intl J. Multiphase Flow 16, 567-578.

South, M. J. \& Hooper, A. P. 1999 Linear growth in two-fluid plane Poiseuille flow. J. Fluid Mech. 381, 121-139.

Straughan, B. 1998 Explosive Instabilities in Mechanics. Springer.

Straughan, B. 2001 Surface-tension driven convection in a fluid overlying a porous layer. J. Comput. Phys. 170, 320-337.

Straughan, B. 2002 Effect of property variation and modeling on convection in a fluid overlying a porous layer. Intl J. Numer. Anal. Meth. Geomech. 26, 75-97.

Straughan, B. 2004 The Energy Method, Stability, and Nonlinear Convection, 2nd edn. Springer.

Stuart, J. T. 1963 Hydrodynamic stability. In Laminar Boundary Layers (ed. L. Rosenhead), pp. 492-579. Oxford University Press.

Suchomel, B. J., Chen, B. M. \& Allen, M. B. 1998 Network model of flow, transport and biofilm effects in porous media. Transport Porous Med. 30, 1-23.

TAYLOR, G. I. 1971 A model for the boundary conditions of a porous material. J. Fluid Mech. 49, 319-326.

Tilley, B. S., Bankoff, S. G. \& Davis, S. H. 1994a Shear and interfacial instabilities in oil-water flow in an inclined channel. Chem. Engng Commun. 141, 41-49.

Tilley, B. S., Davis, S. H. \& BankofF, S. G. $1994 b$ Linear stability theory of two-layer flow in an inclined channel. Phys. Fluids 6, 3906-3922.

Timoshin, S. N. \& Hooper, A. P. 2000 Mode coalescence in a two-fluid boundary-layer stability problem. Phys. Fluids 12, 1969-1978.

Waller, J. \& Schaflinger, U. 1998 Viscous resuspension of a sediment caused by oscillating stratified flows. Acta Mech. 127, 147-153.

Worster, M. G. 1992 Instabilities of the liquid and mushy regions during solidification of alloys. J. Fluid Mech. 237, 649-669.

Zhang, K., Acrivos, A. \& Schaflinger, U. 1992 On the stability in a 2D Hagen-Poiseuille resuspension flow. Intl J. Multiphase Flow 18, 51-63. 